\title{
Spatial frequency and contrast effects on percepts of bistable stroboscopic motion
}

\author{
BRUNO G. BREITMEYER \\ University of Houston, Houston, Texas
}

and

\author{
JAMES G. MAY and MARY C. WILLIAMS \\ University of New Orleans, New Orleans, Louisiana
}

\begin{abstract}
Using alternating frames containing circular patches of vertical sinusoidal gratings, we investigated the effects of spatial frequency and contrast on the perception of end-to-end element and group motion in bistable motion. Experiment 1 showed that for a fixed physical contrast of .33, the percentage of reports of group motion tended to decrease across interstimulus intervals (ISIs) as spatial frequency increased from 1.5 to 6.0 cycles per degree (cpd). In Experiment 2, increasing the contrast of 1.5-cpd elements from .11 to .33 increased the percentage of group motion reports across ISIs. In Experiment 3, we set the physical contrasts of the 1.5- and 3.0-cpd grating patches to match subjectively the apparent contrast of the 6.0-cpd grating set at a physical contrast of .33. With such subjectively equated contrasts, the spatial frequency-dependent trend found in Experiment 1 was either attenuated or eliminated. These results (1) implicate the role of spatial frequency- and contrast-dependent pattern persistence effects on the perception of bistable motion, and (2) in the context of prior studies, suggest that the contribution of this general persistence effect to the perception of bistable motion is independent of the intrinsic spatial scale properties that characterize motion detectors.
\end{abstract}

In two recent studies, Breitmeyer and Ritter (1986a, 1986b) have demonstrated that stimulus factors such as element size, frame duration, and display eccentricity, which systematically affect visual pattern persistence, also have predictable, correlated effects on bistable motion percepts. To generate bistable motion, two stimuli frames, like those depicted in Figure 1, are presented in cyclic alternation. Both frames contain three equispaced, horizontally arrayed elements; however, those of Frame 2, relative to those of Frame 1, are displaced to the right by the center-to-center distance that separates two adjacent elements. When these two frames are alternately flashed for several cycles at variable interstimulus intervals (ISIs), one can observe one of two motion percepts. As originally shown by Pantle and Picciano (1976), at short ISIs one sees the spatially overlapping elements (the middle two) as stationary and the endmost elements as moving back and forth; as the ISI increases, this endto-end element motion is replaced increasingly by a perception of group motion, as the three elements in each frame come to be seen as a group moving back and forth.

Proposals made by Braddick (1980) and Pantle and Petersik (1980) suggest that the perception of end-to-end element motion depends on temporal integration and, thus, on the perceived stationarity of the spatially overlapping elements. The relationship between temporal integration and persistence has been elaborated by Marx and May

Requests for reprints should be directed to B. G. Breitmeyer, Department of Psychology, University of Houston, Houston, TX 77004.
(1983). Breitmeyer and Ritter (1986a, 1986b) extended this proposal by specifically hypothesizing that pattern persistence contributes to the temporal integration process that signals stationarity of the overlapping elements. Their results, as well as those of Petersik and Pantle (1979), showed that stimulus factors that lead to increases of pattern persistence also lead, as expected, to increases of endto-end element motion or, alternately, to decreases of group motion.

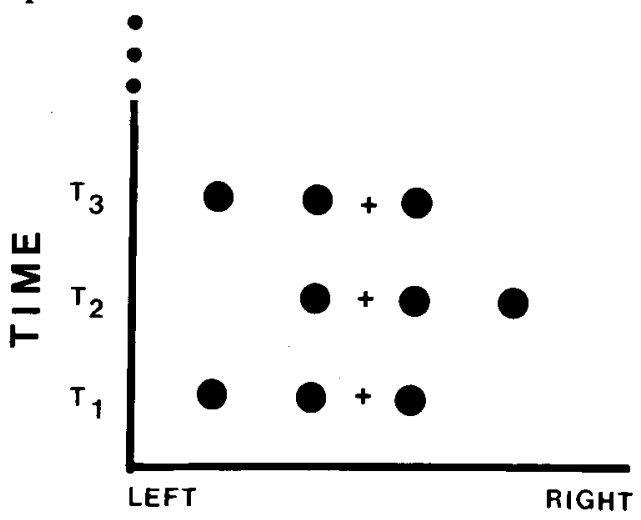

SPACE

Figure 1. A schematic rendering of a bistable motion display sequence. Frame 1, containing three horizontally arrayed elements, is presented at time $T_{1}$. After a variable ISI, Frame 2, containing the same three elements displaced rightward by the center-to-center distance separating two adjacent elements, is presented at time $\mathbf{T}_{\mathbf{2}}$. After the same ISI, Frame 1 again is presented at time $T_{3}$, and so forth for four cycles. 
One such factor is size or spatial frequency. Stimuli that are smaller in size or higher in spatial frequency content have longer persistence, and thus they contribute more to temporal pattern integration (Corfield, Frosdick, \& Campbell, 1978; Di Lollo \& Woods, 1981; Meyer \& Maguire, 1977). Breitmeyer and Ritter (1986a, 1986b) found that decreases in element size indeed led to decreases in the perception of group motion. However, Petersik and Grassmuck (1981) have reported apparently discrepant findings. In one of their experiments, the bistable motion display consisted of two alternating frames, each of which in turn consisted of three cycles of a vertical sinusoidal grating. The two frames were shifted by $360^{\circ}$ relative to each other. Thus two cycles of the gratings overlapped spatially, while one cycle of each grating corresponded to the nonoverlapping, endmost elements of the display sequence. Given such a display, the results showed that the ISI at which the transitions of percept from element to group motion occurred decreased as the spatial frequency increased. According to the persistence hypothesis, the transitional ISI should have increased, because increases of spatial frequency also lead to increases of pattern persistence and integration.

We believe that the following facts are relevant to an explanation of the apparent discrepancy: In the Breitmeyer and Ritter (1986a, 1986b) studies, the center-to-center distance of elements was held constant, and only their size was varied. In the Petersik and Grassmuck (1981) study, both the size of elements (i.e., the width of the dark or light half-cycle of the grating) and their center-to-center separation (i.e., the cycle width) decreased as the spatial frequency increased. Thus, in the Petersik and Grassmuck (1981) study, element size was confounded with the spatial scale factor defined by interelement separation. Hence, decreases of interelement separation as the spatial frequency increased may have been the determining factor in reducing the transitional ISI. Another way of stating this is to say that to decrease interelement separation, as reported by Pantle and Petersik (1980), increases the overall strength of the group motion sensation.

A second factor that affects pattern persistence is stimulus contrast. Using sinusoidal gratings as stimuli, Bowling and Lovegrove (1981) and Bowling, Lovegrove, and Mapperson (1979) showed that visual persistence not only increased as spatial frequency increased, but also decreased as contrast increased. On the basis of the latter result, one would expect subjects to perceive more group motion as the contrast increases. However, Petersik and Pantle (1979), using elements that consisted of dark dots on light backgrounds, found that as the contrast of the dots relative to the background increased, the perception of group motion decreased. As noted below in the description of Experiment 2, these discrepant findings may be related to the fact that Petersik and Pantle's (1979) method for increasing stimulus contrast required a reduction of the intensity of the dots, a procedure that can lead to increases rather than decreases in visual persistence (Bowen, Pola, \& Matin, 1974).
Another relevant finding reported by Bowling et al. (1979) was that increases in spatial frequency produced increases in visual persistence, even when the gratings were equated for subjective contrast. Thus, in their study, the changes in visual persistence as a function of spatial frequency presumably were not due to any correlated changes in apparent contrast. Our Experiment 3, in which gratings were equated for subjective contrast, addressed the implications of these results for the perception of bistable stroboscopic motion.

\section{EXPERIMENT 1}

To investigate the isolated effects of spatial frequency of the elements independently of element size and interelement separation, we devised a display in which each element consisted of a circular patch of a vertical sinusoidal grating whose spatial frequency could be varied, but whose diameter and center-to-center distance from the other element patches were kept constant. Based on the pattern persistence hypothesis, one would predict that across ISIs the reports of group motion should decrease as the spatial frequency composing each element increases.

\section{Method}

Subjects. Three experienced psychophysical observers, including one of the authors, B.B., served as subjects. B.B. was a 40year-old male at the time of the experiments. Subjects C.A. and K.L. were 31-year-old and 27-year-old females. Each subject had at least $1.5 \mathrm{~h}$ of practice at this particular task of distinguishing element from group motion. Practice was given with 4.0 cycles per degree (cpd) elements. This frequency was not used in the experimental sessions. All subjects had natural or corrected visual acuity of 20/20 and were free of other visual anomalies. The 2 female subjects were naive as to the hypotheses under investigation.

Apparatus and stimuli. The stimuli were presented on the face of an oscilloscope (Hewlett-Packard, model 1332a), which was centered in a $20^{\circ}$ field. The surround and the stimulus field were matched in space-averaged luminance $\left(50 \mathrm{~cd} / \mathrm{m}^{2}\right)$ and hue. The stimuli were created by a CRT image generator (Innesfree, Ltd., Picasso) under the control of a minicomputer (Data Translation, Lab Datex). The stimuli consisted of two frames, each with three circular element patches of vertically oriented sine wave gratings. The element patches were arrayed along the horizontal meridian. Each element patch was $2.0^{\circ}$ in diameter, and their center-to-center separation was $2.5^{\circ}$. The fixation point was centered between the rightmost and central element patches of Frame 1, and the leftmost and central element patches of Frame 2 . These two element patches in the two frames thus provided the central, overlapping elements in the entire display sequence. The remaining element patch in each frame (the leftmost in Frame 1 and the rightmost in Frame 2) provided the nonoverlapping, endmost elements of the entire display sequence.

Procedure. A total of 504 trials were run per subject. Of this total, one third (168) of the trials were devoted to each of the following element spatial frequencies: $1.5,3.0$, and $6.0 \mathrm{cpd}$. The grating contrast was .33 at every spatial frequency. Each spatial frequency was tested in a separate block of 168 trials. The order of testing each frequency was counterbalanced, according to a Latin square schedule, across the 3 subjects. Within each block of 168 trials, 24 trials were devoted to each of the following seven interstimulus intervals (ISIs): $10,30,50,70,90,110$, and $130 \mathrm{msec}$. 
SPATIAL FREQUENCY EFFECTS
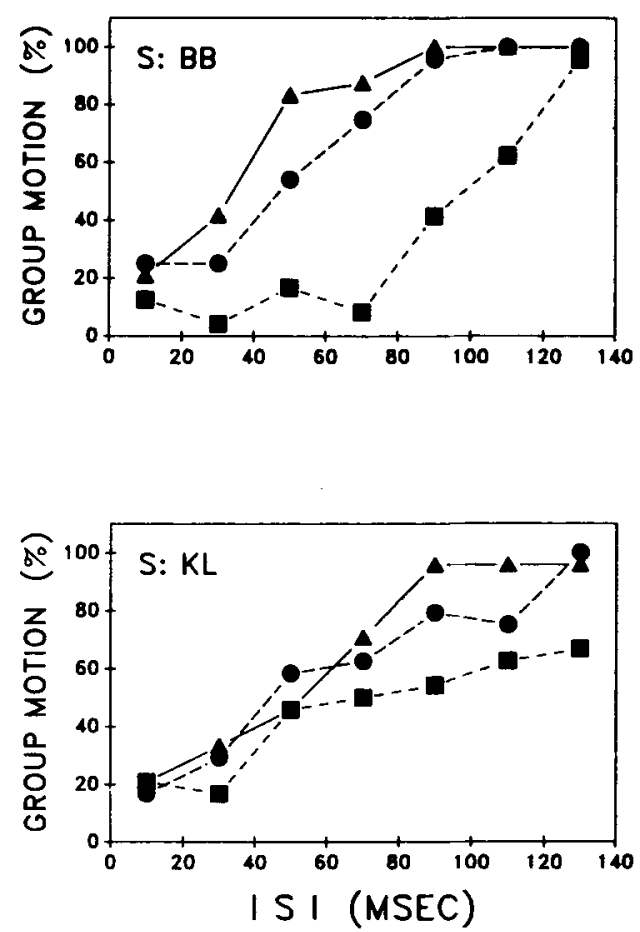

$\Delta=1.5 \mathrm{C} / \mathrm{D}-\mathrm{C}=3.0 \mathrm{C} / \mathrm{D}-\mathrm{D}=6.0 \mathrm{C} / \mathrm{D}$
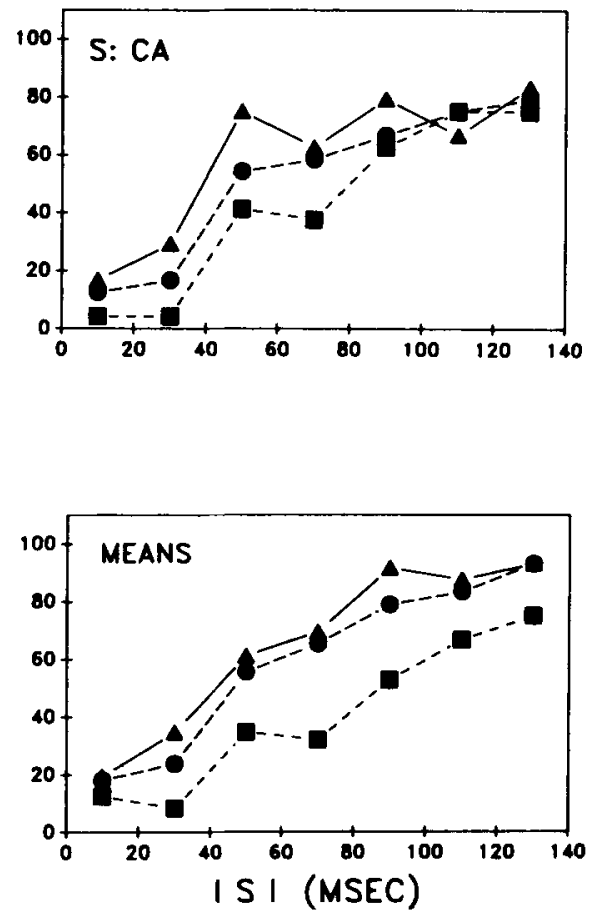

Figure 2. Spatial frequency effects on the percentage of group motion reports as a function of ISI. Results obtained from the individual subjects are shown in the separate panels as indicated; also shown in the lower right panel are the results averaged across subjects. Within each panel, the data are plotted separately for each of the three spatial frequencies, as is indicated above the upper right panel. The contrast of the grating patches comprising the elements was .33.

Subject to this restriction, the ISI order was randomized within a block. On any trial, the two frames (each with a 100 -msec duration) were alternated at one of the randomly chosen ISIs for a total of four cycles. After this presentation, the subjects were asked to indicate whether they perceived group or element motion. Their responses, entered with two keys on a keyboard, were stored in the minicomputer and scored offline at the end of the experiment.

\section{Results and Discussion}

The percentage of group motion reports as a function of ISI and spatial frequency are shown separately for each subject as well as averaged across subjects in Figure 2. For all three subjects the percentage of group motion reports increased along with ISI. This general finding, originally reported by Pantle and Picciano (1976), has been reported in several other studies. Basically, one would expect the temporal integration of the overlapping two element patches to decrease and, thus, group motion reports to increase as the ISI increases. Of greater interest for the present study is the finding that the percentage of group motion reports overall also decreased as the element spatial frequency increased. This was expected on the basis of our pattern persistence hypothesis, and is consistent with the decrease in group motion reports, as the element size decreased, described by Breitmeyer and Ritter $(1986 \mathrm{a}, 1986 \mathrm{~b})$. The combined data were submitted to a $7 \times 3$ repeated measures analysis of variance, which indicated that both main effects [ISI: $F(6,12)=106.94$, $p<.01$; spatial frequency: $F(2,4)=9.29, p<.05$ ] were indeed significant, but that the interaction was not.

\section{EXPERIMENT 2}

Despite the concordance of the above results with our persistence hypothesis, a finding reported by Petersik and Pantle (1979) and alluded to in our introduction seems to be at odds with it. Bowling and Lovegrove (1981) reported that pattern persistence measured with sine wave grating stimuli increased as the contrast of the grating decreased. According to the persistence hypothesis, elements with a lower contrast ought to produce more temporal pattern integration, and, thus, less perception of group motion across ISIs. Petersik and Pantle (1979) reported the opposite result: as the stimulus contrast decreased, the percentage of group motion reports tended to increase rather than decrease. However, in their study, the contrast of the elements was varied by decreasing their intensity from 1.49 to $0.30 \mathrm{~mL}$ against a uniform background of $1.65 \mathrm{~mL}$. From the work of Bowen et al. (1974), we know that visual persistence varies inversely with stimulus intensity. By using progressively less intense elements in order to increase their contrast, Petersik and Pantle (1979) may have inadvertently pitted the 
CONTRAST EFFECTS
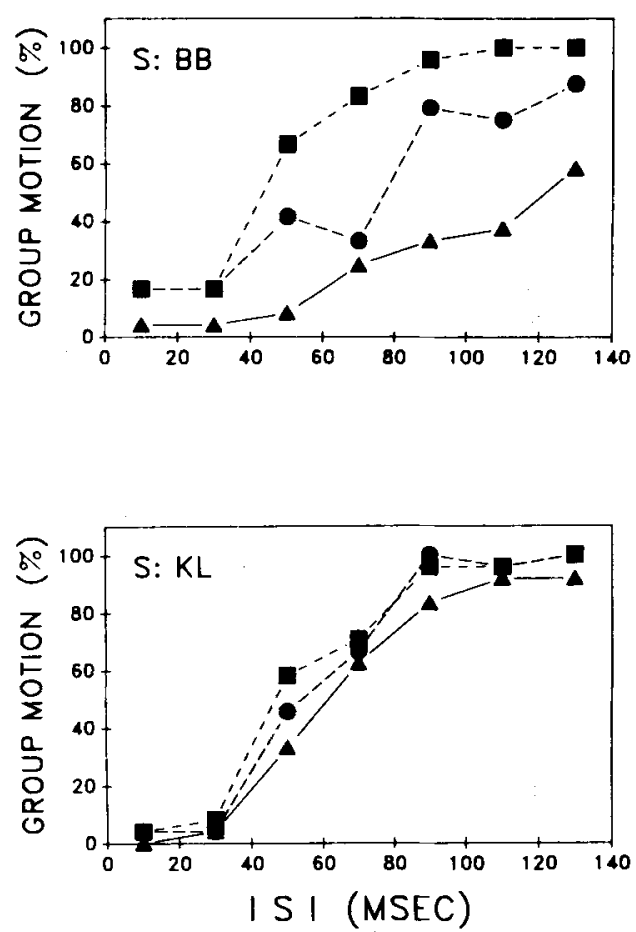
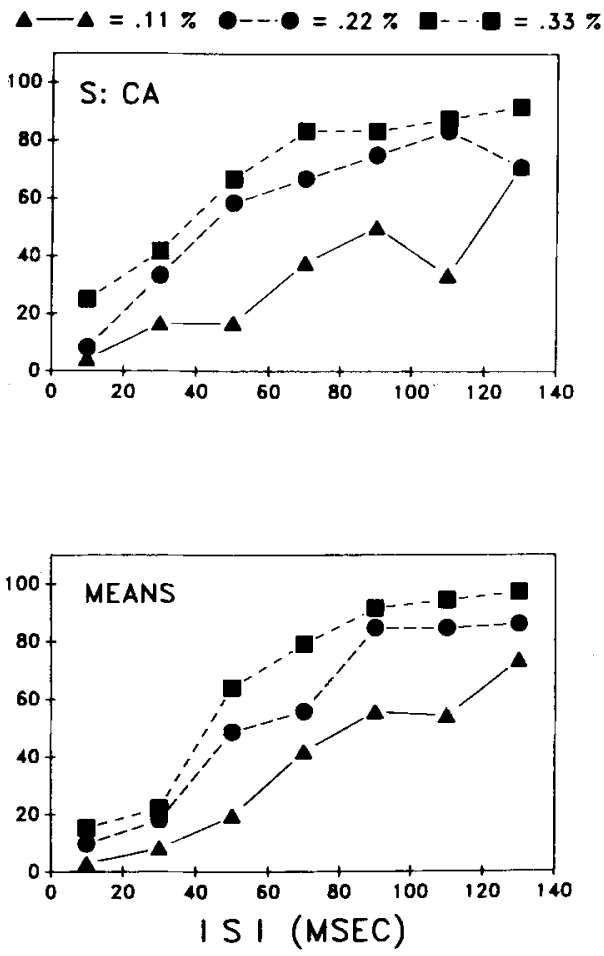

Figure 3. Effects of contrast on the percentage of group motion reports as a function of ISI. Results obtained from the individual subjects are shown in the separate panels as indicated; also shown in the lower right panel are the results averaged across subjects. Within each panel, the data are shown separately for each of the contrasts, as is indicated above the upper right panel. The spatial frequency of the grating patches comprising the elements was $1.5 \mathrm{cpd}$.

effects of stimulus intensity decrements against those of stimulus contrast increments, and thus their procedure may have led to an overall increase in persistence as the element intensity decreased. However, on the basis of Bowling and Lovegrove's (1981) findings as well as our persistence hypothesis, we expect that as the contrast of the sine wave grating patches comprising the elements increases, the persistence will decrease, and thus the percentage of group motion reports will increase across ISIs.

\section{Method}

The subjects and apparatus were the same as in Experiment 1. The stimuli and procedure were also the same, except for the following changes: The spatial frequency of the element gratings was fixed at $1.5 \mathrm{cpd}$. However, the contrast of the gratings could be either .11, .22, or .33. Again, 504 trials were run per subject, with one of the three blocks of 168 trials devoted to each contrast. Order of contrast was counterbalanced across subjects according to a Latin square schedule. Within each block, the same seven ISIs as were used in Experiment 1 were presented in random order. After each trial, the subjects again had to indicate whether they perceived group or element motion.

\section{Results and Discussion}

In Figure 3, the percentage of group motion reports as a function of ISI and contrast is shown separately for each subject and also averaged across subjects. Again, as previously reported, group motion responses increased as ISI increased. More importantly, however, the percentage of group motion reports increased across ISIs as the contrast increased. The latter trend was expected on the basis of our pattern persistence hypothesis. In support of these conclusions, a $7 \times 3$ repeated measures analysis of variance revealed that both main effects [ISI: $F(6,12)=$ $26.57, p<.01$; contrast: $F(2,4)=8.25, p<.05$ ] and their interaction [ISI $\times$ contrast: $F(12,24)=2.53$, $p<.05]$ were significant. The latter significant interaction may be due to ceiling effects imposed on our measure (i.e., the percentage of group motion reports is limited at most to $100 \%$ ). On the other hand, it may reflect a genuine perceptual or sensory effect rather than a mere statistical artifact. For instance, grating contrast may exert its influence in two, possibly interactive, ways on bistable motion. One may consist of the indirect effects of contrast, via pattern persistence, on motion perception; the other, of the direct effects of contrast on the temporal response of motion detectors.

\section{EXPERIMENT 3}

The combined results of Experiments 1 and 2 show that variations in spatial frequency and contrast, by systematically influencing pattern persistence, produce predictable variations in the percentage of group motion reports across ISIs. Since the reduction of physical, and thus apparent, 
contrast increases pattern persistence at any one spatial frequency (Bowling \& Lovegrove, 1981), it is possible that the increase in pattern persistence with increases in spatial frequency is due to the reduction of apparent contrast of gratings at equal physical contrast as their spatial frequency increases (Davidson, 1968). Thus, in Experiment 1 , where the grating patches had the same physical contrast of .33, the increase of pattern persistence as spatial frequency increased from 1.5 to $6.0 \mathrm{cpd}$ may have been due less to spatial frequency per se than to the concomitant decrease of apparent contrast. Accordingly, if the grating patches at the three spatial frequencies used in Experiment 1 are equated for subjective contrast, one would expect less, if any, increase in pattern persistence as the spatial frequency increases. Consequently, according to our persistence hypothesis, the equating of subjective contrasts should decrease or eliminate any spatial frequency-dependent differences in overall percentage of group motion percepts.

This rationale assumes that subjective contrast of suprathreshold gratings varies with spatial frequency in a manner analogous to variations of threshold contrast sensitivity. Such a relationship has been reported by Davidson (1968); however, Georgeson and Sullivan (1975) and Kulikowski (1976) obtained contrast constancy with suprathreshold gratings (i.e., little, if any, variation of apparent contrast with spatial frequency). Procedural differences can account for this discrepancy in findings. Whereas the latter two investigations required contrast matches to be made under steady and prolonged viewing, Davidson's (1968) study required matches to be made under conditions of relatively brief, 500 -msec, stimulus presentations. As noted by Kulikowski (1976), the matches made with briefly presented gratings are not comparable to those made with continuously viewed ones, since the processing times (e.g, integration times; see Breitmeyer \& Ganz, 1977; Brown \& Black, 1976; Legge, Cohen, \& Stromeyer, 1978), are longer for high as compared to low spatial frequencies. Since contrast constancy prevails with gratings matched under continuous viewing, one would expect gratings matched for contrast under this viewing condition to continue to yield increases in visual persistence as their spatial frequency increases. In fact, the results reported by Bowling et al. (1979) confirm this expectation. However, Davidson (1968) found that contrast constancy fails to hold under brief viewing. Hence, for gratings whose apparent contrasts are matched under this latter viewing condition, one might fail to obtain increases in visual persistence as the spatial frequency increases. In our contrast matching, transient viewing was employed.

\section{Method}

The subjects, apparatus, and procedure were as those in Experiment 1; only the stimuli were changed, in the following way: for each subject, the physical contrasts of the 1.5- and 3.0-cpd gratings were individually adjusted until their subjective contrasts matched that of the 6.0-cpd grating whose physical contrast was set at .33. Matches were performed by first flashing the 6.0 -cpd grating for $100 \mathrm{msec}$, and then, after an interval of $1 \mathrm{sec}$, flashing the 1.5- or 3.0-cpd grating for $100 \mathrm{msec}$. The contrast of the latter grating, using a modified method of ascending and descending limits, was increased or decreased stepwise in 3-dB steps until the subject judged its apparent contrast to match that of the 6.0-cpd grating. The physical contrast of the 1.5 - or 3.0-cpd grating that produced subjective contrast matches to the 6.0 -cpd grating was defined as the average value of four ascending and four descending limits. In line with Davidson's (1968) results, contrast constancy did not prevail under these transient viewing conditions. For all three subjects, the physical contrasts of the 3.0- and 1.5-cpd gratings required to produce a perceived contrast match with the 6.0-cpd grating were consistently and noticeably lower than .33 , with the 1.5 -cpd grating tending to require somewhat less physical contrast than the 3.0cpd grating.

\section{Results and Discussion}

Figure 4 shows the percentage of group motion reports as a function of ISI and spatial frequency separately for each subject and, additionally, averaged across subjects. Again, as in Experiment 1, the percentage of group motion reports increased as the ISI increased. However, when comparing results shown in Figure 4 with those in Figure 2, one can see that the spatial frequency-dependent effects on group motion percepts obtained in Experiment 1 are attenuated or eliminated in Experiment 3. For the combined data obtained in Experiment 3, a $7 \times 3$ repeated measures analysis of variance revealed that only the main effect of ISI $[F(6,12)=141.55, p<.01]$ was significant. Neither the main effect of spatial frequency nor its interaction with ISI was significant. Hence, these results confirmed our expectation that the spatial frequencydependent changes in pattern persistence and, thus, in the strength of the group motion sensation would be eliminated when subjective contrast was equated across spatial frequencies.

\section{GENERAL DISCUSSION}

When, as in Experiment 1, the spatial scale factors of interelement separation and of element diameter are kept constant and only the spatial frequency composition of the elements is varied, the resulting changes in group motion responses to a bistable motion display accord with our pattern persistence hypothesis. In particular, it was shown that increases in the spatial frequency of the constant-contrast, sine wave grating that constituted each element led to a decrease of group motion responses across ISIs. A decrease in group motion or, alternately, an increase of element motion reports is the result of the temporal pattern integration of the two overlapping elements, which leads to their being perceived as stationary. Since pattern persistence contributes to temporal integration (Di Lollo \& Woods, 1981), one would indeed expect that increases in spatial frequency, which, at constant physical contrast, produce increases in pattern persistence (Cor- 
SPATIAL FREQUENCY EFFECTS
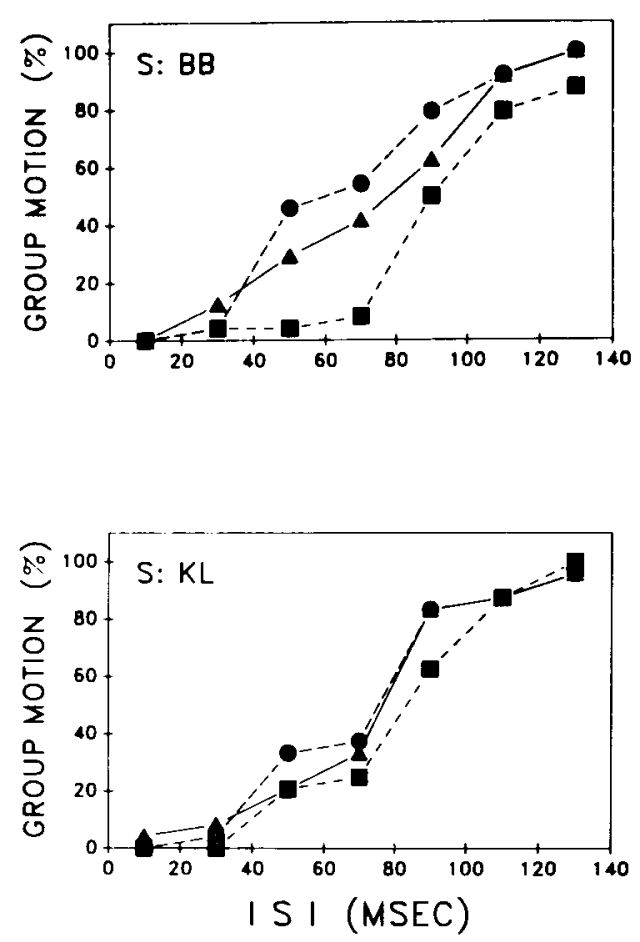

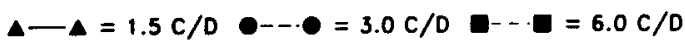
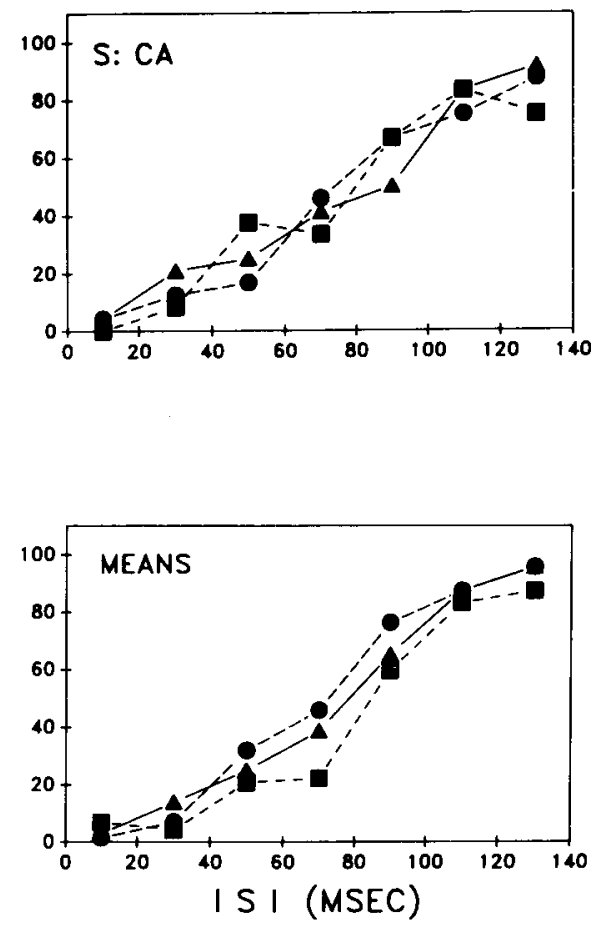

Figure 4. Spatial frequency effects, under conditions of subjectively matched contrasts, on the percentage of group motion reports as a function of ISI. Results obtained from the individual subjects are shown in the separate panels as indicated; also shown in the lower right panel are the results averaged across subjects. Within each panel, the data are plotted separately for each of the three spatial frequencies, as is indicated above the upper right panel.

field et al., 1978; Meyer \& Maguire, 1977), should lead to a reduction in group motion reports.

Nonetheless, spatial scale factors such as interelement separation are also important in the perception of bistable motion. In particular, Pantle and Petersik (1980) demonstrated clearly that reducing the center-to-center separation of elements of constant width and height produced more group motion responses across ISIs. Hence, spatial frequency composition and interelement separation seem to contribute independently, but not necessarily equally, to bistable motion perception. This is made evident by Petersik and Grassmuck's (1981) findings. In one of their conditions, the stimuli consisted of three cycles of a vertical sinusoidal grating shifted back and forth from one frame to the other by a phase angle of $360^{\circ}$. Here, increases in spatial frequency, which, as a result of greater pattern persistence, would lead to increases in the transitional ISI, were covaried with decreases in interelement separation, which would lead to decreases in the transitional ISI. Yet these two apparently equal effective trends did not cancel each other out; instead the latter trend was found to be dominant. Thus, it appears that spatial scale factors such as interelement separation are more important to the perception of bistable motion than are spatial frequency composition or the size of elements. The fact that spatial frequency compo- sition does affect the perception of bistable stroboscopic motion, however, can be inferred from additional results, obtained by Petersik and Grassmuck (1981) under a second condition in which they employed square wave instead of sine wave gratings. Since a spatial square wave is the sum of the fundamental sine wave and the odd higher harmonics, the latter higher spatial frequency components would tend to increase the total pattern persistence of the square wave grating. According to our persistence hypothesis, this should favor end-to-end element motion percepts and thus increase the ISI at which transitions from element to group motion percepts occur, a finding reported by Petersik and Grassmuck (1981). Experiment 2 showed that decreases in the physical contrast of the element gratings leads to decreases in group motion reports. This result is also consistent with our pattern persistence hypothesis, since decreases in contrast produce increases in pattern persistence (Bowling \& Lovegrove, 1981; Bowling et al., 1979). The contrary finding of Petersik and Pantle (1979) noted above can be explained by their procedure for increasing the contrast, which involved a reduction in the element luminance relative to a uniform background of constant higher luminance. It is known that visual persistence increases with decreases in stimulus luminance (Bowen et al., 1974). On the reasonable but as yet untested assumption that the effects on visual persis- 
tence of decreasing the stimulus luminance dominate the countervailing effects of increasing the stimulus contrast, one would expect less group motion across ISIs, as found by Petersik and Pantle (1979).

Experiment 3 showed that for elements consisting of grating patches of variable frequency but equal subjective contrast, the spatial frequency-dependent changes in group motion reports found in Experiment 1 are attenuated, if not eliminated. This indicates that the increases in pattern persistence accompanying increases in the spatial frequency of gratings of equal physical contrast may largely be due to the reduction of apparent contrast at higher spatial frequencies during brief stimulus presentations (Davidson, 1968). As shown by Bowling et al. (1979), gratings equated for apparent contrast under continuous viewing continue to yield increases in pattern persistence under transient viewing, as spatial frequency increases. However, when both the matching of apparent contrast and the measurement of persistence occur under the same brief viewing condition, one might expect the elimination of the spatial frequency-dependent changes in visual persistence. Current research in our laboratories is testing this possibility.

The present results and interpretations may also be relevant for our understanding of motion processes investigated with random-dot cinematograms (RDCs) (Julesz, 1971). Braddick (1974) showed that the upper limit of spatial displacement (Dmax) that still allowed the perception of coherent motion in two stroboscopically flashed RDC frames was relatively low, on the order of $15^{\prime}$; hence the designation of short-range motion. This upper short-range limit is much smaller than the upper limit of several degrees that characterizes the long-range motion process activated with classical stimuli (Zeeman \& Roelofs, 1953). As is frequently the case (see e.g. Baker \& Braddick, 1982; Bischof \& Groner, 1985), displacements in RDCs are usually in integral pixel or dot steps. When the dot density is high-say, .5-the probability that any two dots from successive frames of the RDC display will overlap spatially is also high-in this particular case, .5. This would lead to $50 \%$ of the dots integrating temporally at short ISIs, which, relative to the motion signal generated by the remaining $50 \%$ of the nonoverlapping dots, constitutes a substantial counteracting stationarity signal. This inertial signal could contribute to the low Dmax value with high dot-density RDCs. Decreases in the dot density, which in turn decrease the proportion of dots overlapping spatially in successive frames, would produce a weaker stationarity signal at short ISIs. Hence, Dmax ought to increase as dot density decreases, a result reported by Ramachandran and Anstis (1983) and Bischof and Groner (1985).

\section{REFERENCES}

BAKER, C. L., JR., BradDICK, O. (1982). The basis of area and dot number effects in random dot motion perception. Vision Research, 22, 1253-1259.

BischoF, W. R., \& Groner, M. (1985). Beyond the displacement limit:
An analysis of short-range processes in apparent motion. Vision Research, 25, 839-847.

Bowen, R. R., PolA, J., \& MATIN, L. (1974). Visual persistence effects of flash luminance, duration, and energy. Vision Research, 14, 295-303.

Bowling, A., \& Lovegrove, W. (1981). Two components of visual persistence: Effects of orientation and contrast. Vision Research, 21, 1241-1251.

Bowling, A., Lovegrove, W., \& Mapperson, B. (1979). The effect of spatial frequency and contrast on visual persistence. Perception, 8, 529-539.

Braddick, O. (1974). A short-range process in apparent motion. Vision Research, 14, 519-527.

Braddick, O. (1980). Low-level and high-level processes in apparent motion. Philosophical Transactions of the Royal Society, Series B, 290, 137-151.

Breitmeyer, B. G., \& Ganz, L. (1977). Temporal studies with flashed gratings: Inferences about human transient and sustained channels. Vision Research, 17, 861-865.

Breitmeyer, B. G., \& RitTer, A. (1986a). The role of visual persistence in bistable stroboscopic motion. Vision Research, 26, 1801-1806.

BREITMEYER, B. G., RitTER, A. (1986b). Visual persistence and the effect of eccentric viewing, element size, and frame duration on bistable stroboscopic motion percepts. Perception \& Psychophysics, 39, 275-280.

Brown, J. L., BLACK, J. E. (1976). Critical duration for resolution of acuity targets. Vision Research, 16, 309-315.

Corfield, R., Frosdick, J. P., \& Campbell, F. W. (1978). Grey-out elimination: The roles of spatial waveform, frequency and phase. $\mathrm{Vi}$ sion Research, 18, 1305-1311.

DAvidson, M. L. (1968). Perturbation approach to spatial brightness interaction in human vision. Journal of the Optical Society of America, 58, $1300-1309$.

Di LolLo, V., \& Woods, E. (1981). Duration of visible persistence in relation to range of spatial frequencies. Joumal of Experimental Psychology: Human Perception \& Performance, 7, 754-769.

Georgeson, M. A., Suluivan, G. D. (1975). Contrast constancy: Deblurring in human vision by spatial frequency channels. Jourmal of Physiology, 232, 627-656.

Julesz, B. (1971). Foundations of cyclopean perception. Chicago: University of Chicago Press.

KULIKowskı, J. J. (1976). Effective contrast constancy and linearity of contrast sensation. Vision Research, 16, 1419-1431.

LegGe, G. E., Cohen, M. A., \& Stromeyer, C. F. (1978). Spatialfrequency masking with briefly pulsed patterns. Perception, 7, 161-166.

MARX, M. S., \& MAY, J. G. (1983). The relationship between temporal integration and persistence. Vision Research, 23, 1101-1106.

Meyer, G. E., \& Maguire, W. M. (1977). Spatial frequency and the mediation of short-term visual store. Science, 198, 524-525.

Pantle, A. J., \& Petersix, J. T. (1980). Effects of spatial parameters on the perceptual organization of a bistable motion display. Perception \& Psychophysics, 27, 307-312.

Pantle, A., \& Picciano, L. A. (1976). A multistable movement display: Evidence for two separate motion systems in human vision. Science, 193, 500-502.

Petersik, J. T., \& Grassmuck, J. (1981). High fundamental spatial frequencies and edges have different perceptual consequences in the "group/end-to-end" movement phenomenon. Perception, 10 375-382.

Petersik, J. T., \& Pantle, A. J. (1979). Factors controlling the competing sensations produced by a bistable stroboscopic motion display. Vision Research, 19, 143-154.

Ramachandran, V. S., \& ANSTIS, S. M. (1983). Displacement thresholds for coherent apparent motion in random-dot patterns. $V i$ sion Research, 23, 1719-1724.

ZEEMAN, W. P., RoELoFs, C. O. (1953). Some aspects of apparent motion. Acta Psychologica, 9, 159-181.

(Manuscript received May 26, 1987; revision accepted for publication June 8, 1988.) 